\title{
Extinction risk controlled by interaction of long-term and short-term climate change
}

Authors: Gregor H. Mathes ${ }^{1,2^{*}}$, Jeroen van Dijk ${ }^{1}$, Wolfgang Kiessling ${ }^{1}$, Manuel J. Steinbauer ${ }^{2,3}$

\section{Affiliations:}

${ }^{1}$ Friedrich-Alexander University Erlangen-Nürnberg (FAU), GeoZentrum Nordbayern, Department of Geography and Geosciences, 91054 Erlangen, Germany

${ }^{2}$ University of Bayreuth, Bayreuth Center of Ecology and Environmental Research (BayCEER), 95447 Bayreuth, Germany

${ }^{3}$ University of Bergen, Department of Biological Sciences, 5006 Bergen, Norway

${ }^{*}$ Corresponding author. Email: gregor.mathes@uni-bayreuth.de

Summary: Assessing extinction risk from climate drivers is a major goal of conservation science. Few studies, however, include a long-term perspective of climate change. Without explicit integration, such long-term temperature trends and their interactions with short-term climate change may be so dominant that they blur or even reverse the apparent direct relationship between climate change and extinction. Here we evaluate how observed genuslevel extinctions of arthropods, bivalves, cnidarians, echinoderms, foraminifera, gastropods, mammals, and reptiles in the geological past can be predicted from the interaction of longterm temperature trends with short-term climate change. We compare synergistic palaeoclimate interaction (i.e. a short-term change on top of a long-term trend in the same direction) to antagonistic palaeoclimate interaction such as long-term cooling followed by short-term warming. Synergistic palaeoclimate interaction increases extinction risk by up to 40\%. The memory of palaeoclimate interaction including the climate history experienced by ancestral lineages can be up to 60 myr long. The effect size of palaeoclimate interaction is similar to other key factors such as geographic range, abundance, or clade membership. Insights arising from this previously unknown driver of extinction risk might attenuate recent predictions of climate-change induced biodiversity loss. 


\section{Main Text}

Biodiversity and ecosystems are critically endangered by current climate change ${ }^{1,2}$. Reliable assessments of extinction risk are thus essential for the effective protection of biodiversity ${ }^{3,4}$. Whereas several assessments categorize extinction risk from climate change ${ }^{5,6}$, relatively few studies make use of the rich information on past biotic responses to climate changes provided by the fossil record ${ }^{7-9}$. Palaeontologically informed models have proven to be powerful in discerning biotic factors that determine future extinction vulnerability ${ }^{10-12}$. Numerous reports demonstrate that the impact of climatic changes increases with distance from the climatic conditions species are adapted to ${ }^{13-15}$. Additionally, a strong link between temperature stress and extinction risk is also known from the fossil record ${ }^{16-18}$. However, one potentially crucial factor of extinction dynamics, the interaction of long-term temperature trends with short-term temperature change, has not been investigated until now.

Here we quantify how the interaction of long-term temperature trends with short-term temperature change can affect temperature-related extinction probabilities. We expect temperature change to be more critical when it adds to previous trends in the same direction (i.e., synergistic interactions), because taxa then face conditions increasingly different from previous adaptations. To the contrary, current anthropogenic warming occurs after a 40 million year cooling trend, raising the possibility that many modern clades are increasingly exposed to climates they experienced during their origination. Such change may be less harmful. Understanding the effect of this palaeoclimate interaction could hence provide crucial insights into extinction mechanisms and lead to improved mitigation efforts for biodiversity under current climate change.

We analysed eight fossil clades, both marine and terrestrial, each containing more than 400 genera. We implemented generalised linear mixed effect models with binomial family error (GLMMs) ${ }^{19}$ to explain how survival and extinction on genus-level is affected by 
palaeoclimate interaction (Fig. 1). Besides quantifying effect size, we also estimated the temporal memory of this effect. We then compared these models containing information about both long-term temperature trends and short-term changes to models including shortterm temperature changes only using model selection ${ }^{20}$.

\section{Results}

Palaeoclimate interactions improved model performance in 7 out of 8 clades when compared to models including short-term temperature changes only, based on Akaike’s Information Criterion (AIC) (Fig. 2). Only mammals showed a deteriorating model performance for shortterm cooling when palaeoclimate interaction was included.

Short-term climate change adding to a previous temperature trend in the same direction (synergistic palaeoclimate interaction) increased extinction risk in all significant models (Fig. 3, Extended Data 1). Model predictions showed an increase in extinction risk between $10 \%$ and $40 \%$ after such a synergistic palaeoclimate interaction for arthropods, bivalves, foraminifera, mammals, and reptiles. The synergistic interaction of long-term cooling with short-term cooling yielded the most severe impact on extinction risk with a $40 \%$ increase for mammals and 33\% for foraminifera.

We observe a negative relationship between extinction risk predicted by the interaction models and the duration of genera (Fig. 4). The effect of palaeoclimate interaction on extinction is strongest for clades with short-duration genera, whereas clades with greater durations of genera experienced a lower change in extinction risk. The climatic history of each genus accounted for $37 \%$ variation of median and $40 \%$ of mean duration (Adjusted $\mathrm{R}^{2}$; based on F-statistics).

The extinction risk of marine and terrestrial taxa is dependent on temperature trends extending over a period of 5 to 60 million years or up to 10 geological stages. For each genus within a clade, we calculated up to 10 long-term temperature trends interacting with short-term 
temperature change. We subsequently determined the period of time, where the interaction resulted in the strongest change in intrinsic extinction risk (i.e. temporal memory, Extended Data 2). Some clades such as arthropods, foraminifera, and reptiles are more responsive to temperature changes with geologically short durations (5 to 24 million years), while bivalves, cnidarians, echinoderms, gastropods, and mammals respond more strongly to interactions with geologically long climate trends (24 to 60 million). The temporal memory of the interaction effect was independent from the durations of genera (Extended Data 3). Null models of temperature-independent extinction/survival processes were used to test the robustness of our analytical results (Extended Data 4, see Methods section). These simulations resulted in negligible changes of intrinsic extinction risk suggesting that we reveal biologically meaningful patterns.

\section{Discussion}

We show that palaeoclimatic interactions can significantly elevate the temperature-related extinction risk of organisms, and that this increase is negatively linked to the durations of fossil genera. Our results are consistent with previous findings, revealing profound impacts of temperature change on extinction risk ${ }^{16-18}$. However, the effect of palaeoclimate interaction is so strong, that both neontological and palaeontological studies may either overestimate or underestimate the impact of short-term temperature change when ignoring the interaction with long-term trends. Incorporating long-term temperature trends, and thus climate history, generally improves model performance and can increase inferred extinction risk by up to $40 \%$ when compared to antagonistic palaeoclimate interaction. Considering the vast amount of additional biotic and abiotic factors that contribute to extinction risk ${ }^{21}$, the explanatory power of palaeoclimate interaction compared to traditional models is remarkable. Our results indicate that the effect size of palaeoclimate interaction is on par with other key factors such as geographic range $\mathrm{e}^{8,11,22}$, abundance $\mathrm{e}^{23,24}$, or clade membership $\mathrm{p}^{7,21,25}$. 
Hypothetically, the effect of synergistic temperature change on extinction risk can be caused by three (or potentially more) ecological mechanisms: Niche conservatism ${ }^{13,26}$, migration $\operatorname{lags}^{27,28}$, and cascading effects ${ }^{14,15}$. All three mechanisms are based on the concept of niche stability over geological time scales and imply that synergistic temperature changes successively move taxa out of their adaptation space. An additional short-term perturbation in the same direction as the trend is thus expected to increase extinction risk. Although variable among major clades, evidence for niche stability abounds in the fossil record ${ }^{26,29}$. Understanding the mechanisms of palaeoclimate interaction is particularly relevant for palaeontologically informed models to assess extinction risk under current climate change. Previous models ${ }^{7-9}$ were calibrated in a trend of long-term cooling during the Paleogene and Neogene. Within this nearly monotonic long-term cooling, only two types of palaeoclimate interactions can occur: Antagonistic short-term warming on top of a cooling trend and synergistic short-term cooling on top of a cooling trend, with the latter being more common. Based on our results, previous predictions may overestimate the extinction risk of modern taxa under current climate change (Fig. 5).

Directly projecting palaeoclimate interactions into the near-time future is hampered by insufficient knowledge of underlying mechanisms and potential scaling effects. Our analysis covers long geological time spans and does not take short-scale climatic variations into account. Hence, assessing the effect of palaeoclimate interaction on extinction risk over shorter timescales (millennia or even centuries) should be the focus of future research. We show that the effect of palaeoclimate interaction can prevail over millions of years. However, niche stability has been ascertained over different spatial and temporal scales, as well as taxonomical hierarchies ${ }^{13,26,30}$. Likewise, our hypothesis that synergistic temperature changes successively move taxa out of their adaptation space and hence increase their extinction risk remains valid over ecological time spans such as centuries ${ }^{13-15,27,28}$. We thus expect palaeoclimate interaction to be a key mechanism of extinction risk over shorter time scales as 
well. Besides contributing to improved baseline estimates of vulnerability for the future, our findings also provide insights into the past. As the palaeoclimatic history a clade has experienced is a strong determinant of its fate, studies about extreme climate change events in the geological past should take climatic developments before such an event into account. Hyperthermal events represent natural examples of abrupt climate changes ${ }^{31}$. These abrupt climate changes may coincide with severe mass extinctions ${ }^{4,32}$, but not necessarily so. The Palaeocene-Eocene Thermal Maximum (PETM), for example, was not associated with profound extinctions ${ }^{31}$. The effect of palaeoclimate interaction could provide an explanation for this conundrum, as short-term warming in the PETM follows a general long-term cooling trend in the late Cretaceous (Fig. 5). This is particularly important, as the PETM is often used as an analogue of anthropogenic climate change and for testing climate models ${ }^{31}$. Major biotic turnovers during short-term cooling at the Eocene-Oligocene boundary ${ }^{33}$, on the other hand, were potentially amplified by a previous long-term cooling trend throughout the Paleogene (Fig. 5).

By providing insights into an understudied key mechanism of extinction processes, our findings may hence facilitate the interpretation of temperature-driven extinction events. Without explicit integration, palaeoclimate interaction could blur or even reverse apparent extinction patterns and prevent useful predictions for future scenarios, as has been shown for other complex ecological interactions ${ }^{34,35}$. Current assessments of extinction vulnerability under future climate change neither include palaeoclimatic interactions nor a long-term temperature history of the studied taxa. Given the long-term cooling that most living taxa have experienced in their duration, extinction risk under future warming might be less severe than these assessments predict. 


\section{Methods}

\section{$\underline{\text { Fossil data }}$}

Occurrences of post-Cambrian arthropods, bivalves, gastropods, reptiles (including birds and dinosaurs), cnidarians, and echinoderms were downloaded from the Paleobiology Database (PaleoDB, paleobiodb.org) on 21 September 2020. We further downloaded occurrence data for Cenozoic mammals from the New and Old Worlds Database (NOW, www.helsinki.fi/science/now/) on 23 September 2020. We favoured NOW over the PaleoDB for mammal data, as mammal occurrences are continuously controlled and revised by NOW advisory board members. Stratigraphic range data for post-Cambrian large benthic foraminifera were compiled from a comprehensive reference work on larger foraminifera ${ }^{36}$ and merged with occurrence data downloaded for planktonic foraminifera from the Neptune Database (nsb-mfn-berlin.de) accessed on 23 September 2020. For each download, we included all occurrences with current latitude and longitude, the actual taxonomic name by which the occurrence was identified, and additional information about the taxonomic classification.

We included marine invertebrate clades (cnidarians, gastropods, bivalves, echinoderms, foraminifera, arthropods) known for their good fossil record ${ }^{37}$ in our analysis and added terrestrial vertebrate animals (reptiles, mammals) to ensure coverage of all possible lifestyles and habitats. Each clade contained more than 400 genera, adding up to more than 14, 900 analysed genera in total after applying our cleaning protocol (Extended Data 5).

All analyses were conducted at the genus level. This taxonomic level is a compromise between uncertainty in the species level taxonomy of fossils and data loss at coarser taxonomic resolutions ${ }^{38}$. To ensure uniformity of data sets, we applied a standardised cleaning protocol to all of them including removal of genera ranging to the Recent, uncertain taxonomical ranks, duplicates in bins, single-collection, and single-reference taxa as well as 
missing higher-level taxonomy. We then transformed occurrence data into ranges congruent to a time series with one single origination and extinction event for each genus using the $\mathrm{R}$ package divDyn ${ }^{39}$.

We subsequently binned the data into one of 80 geological stages ${ }^{40}$, ranging from the Ordovician to the Pleistocene. The Holocene was excluded from the analysis. Additionally, taxa confined to a single stage (singletons) were excluded for all data sets as they tend to produce undesirable distortions of the fossil record ${ }^{41}$.

\section{Climate proxy data}

To reconstruct temperature change over time, we used the tropical whole surface water (mixed layers $<300 \mathrm{~m}$ deep) oxygen isotope dataset from a compilation of marine carbonate isotopes ${ }^{42}$. The $\delta^{18} \mathrm{O}$ values of well-preserved calcareous shells are often considered as the best available temperature proxy for the fossil record ${ }^{43}$.To reduce bias while calculating palaeotemperature from the raw $\delta^{18} \mathrm{O}$ values, we followed the data processing of Reddin et al. ${ }^{37}$. This includes adjustments for the secular trend in oxygen isotopic composition of seawater using the equation: $\delta^{18} \mathrm{O}_{\mathrm{pw}}(\%)=\mathrm{t}^{2}+0.0046 \% \mathrm{t}$, with $\mathrm{pw}$ being Phanerozoic seawater in standard mean ocean water (SMOW) and $t$ being age in Ma, as well as averaging of tropical and subtropical records. We subsequently binned temperature data based on isotope values to geological stages to provide global mean temperature for each of the 80 stages. We emphasise that the interpretation of $\delta^{18} \mathrm{O}$ values in deep time is a subject of considerable debate ${ }^{42-46}$. Throughout our data processing, we follow the argumentation of Veizer and Prokoph ${ }^{42}$, inferring a secular increase in seawater $\delta^{18} \mathrm{O}$ values due to changes in how surficial oxygen reservoirs are exchanged with the vastly larger oxygen reservoir in crust and mantle silicates. 


\section{Generalised linear mixed effect models}

All analyses were carried out in $\mathrm{R}^{47}$, using $\mathrm{R}$ version 4.0.2. We used the lme4 package ${ }^{48}$ to perform the analysis, and the ggplot2 package $^{49}$ to visualise results.

We quantified the effect of temperature change interacting with past temperature trends on extinction risk using generalised linear mixed effect models with a binomial family error

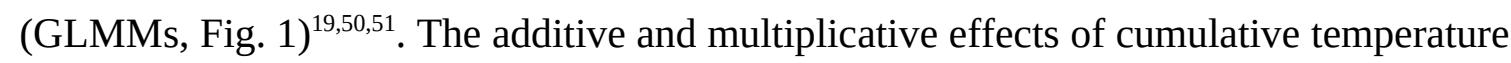
change were tested against the probability of extinction in each time interval in the form: glm (extinct $\sim \Delta \mathrm{T}_{\text {trend }}: \Delta \mathrm{T}_{\text {change }}+($ Stage $\mid$ Genus), family $=$ binomial).

In a first step, we aligned the climate proxy data with the fossil data. Each genus was hence represented as a time series of repeated survivals followed by one extinction event. Each observation within this time series was associated with a specific magnitude in temperature change compared to the previous observation ( $\left.\Delta \mathrm{T}_{\text {change }}\right)$, assessed by using the slope of a linear regression of these two observations. Additionally, we computed long-term temperature trends $\left(\Delta \mathrm{T}_{\text {trend }}\right)$. Each of these long-term trends was evaluated by the slope of a linear regression across temperature estimates of 1-10 time intervals prior to a focal interval. In this way, $\Delta \mathrm{T}_{\text {change, }}$ defined as the change in temperature compared to the previous stage, was excluded from $\Delta \mathrm{T}_{\text {trend, }}$ enabling the independent calculation of palaeoclimate interaction. Each of the trends covered a successively growing time of temperature history: Trend.st1 ranged one stage back, trend.st2 two stages, ..., trend.st10 ten stages (Fig. 1).

We controlled for the fact that observations on the same genus are non-independent by including genus identity as a random effect. By also setting stage as a random effect, we allowed for a random slope of each stage within each genus with correlated intercept, accounting for the temporal structure of the data ${ }^{19,52}$. For each fossil clade, we calculated up to 10 GLMMs for cooling-cooling and warming-warming interaction, respectively. Within these 
models, $\Delta \mathrm{T}_{\text {change }}$ was fixed as the short-term temperature change leading up to the extinction event of a genus. $\Delta \mathrm{T}_{\text {trend }}$ varied for each model based on long-term trends.

The model performance of GLMMs with different long-term trends were compared using Akaike’s information criterion (AIC) ${ }^{20}$, and for each group one final model was selected based on AIC for further analytical evaluation. Results remained the same when using Bayesian Information Criteria for model performance. We additionally determined the significance of palaeoclimate interaction on extinction risk for each model using maximum likelihood.

\section{Model comparison}

Palaeontological studies on temperature-related extinctions usually rely on the temperature change from the previous geological stage to the focal stage, defined here as short-term change. To test if models improve when long-term temperature trends are included, we compared GLMMs including short-term change only to GLMMs including the palaeoclimatic interaction of short-term change with long-term trends. For models taking only short-term change into account, we used the same data processing as aforementioned but excluded longterm trends from the final model:

glm (extinct $\sim \Delta \mathrm{T}_{\text {change }}+$ (Stage $\mid$ Genus), family = binomial) .

\section{Quantifying change in intrinsic extinction risk}

We extracted the results of each final GLMM using the predict() function. To quantify the effect of cumulative temperature change on extinction risk, we transformed the results from odds ratio to probability. We compared the effect of cooling-cooling interaction on extinction risk with every other possible interaction of short-term cooling, and vice versa the effect of warming-warming interaction with every other possible interaction of short-term warming (Extended Data 1). To do so, we used Wilcoxon rank sum tests with continuity correction to compare effect sizes. Results were recorded including 95\% Wald confidence intervals derived from the Wilcoxon rank sum tests. 
Finally, we compared these results to the simulated effect range of null models to test if results could be produced by a random structure (see Null models below). This significance testing approach was implemented with Wilcoxon rank sum tests with continuity correction comparing simulated distributions with results based on observed data.

\section{Assessing temporal memory of past climate change effects}

By calculating one GLMM model for each long-term temperature trend ( $\left.\Delta \mathrm{T}_{\text {trend }}\right)$, we were able to choose the trend explaining the most variation of extinction risk in interaction with shortterm change. The model with the lowest AIC also showed the highest effect of past temperature change on extinction risk. Effect size decreased with increasing AIC of the remaining models, enabling determination of the temporal memory of the effect based on the AIC (Extended Data 2).

\section{Null models}

Estimating the parameters of a statistical model is a key step in statistical analyses. However, fitting fixed-effect parameters of a GLMM can lead to biased statistical inference ${ }^{19}$. To avoid this bias, we applied our model structure to randomly generated data (Extended Data 4). We then used the results of these 'random' models as a distribution to compare with empirical results. This approach enabled the determination of type I error rates of our models and the probability of obtaining results as strong as those observed from a random structure with intrinsic biases of non-fossil data, such as serial autocorrelation of climate proxy data. Incorporating this two-tailed null hypothesis testing in addition to the maximum likelihood framework renders our statistical inference highly robust and reliable ${ }^{53}$. This multiple testing approach is conservative at the cost of increased type II error rates ${ }^{54}$. Our analysis therefore tends to discard palaeoclimate interactions with moderate intensity as insignificant, even when these interactions had a measurable effect on extinction risk. 
Null models started with generating First Appearance Datums (FAD) using a random number generator. We generated 3000 FADs from a uniform distribution. Last Appearance Datums

(LAD) were assigned by drawing a number from the durations of all genera with replacement from our observed data and subtracting it from the FAD. Distributions of the generated datasets therefore simulated observed conditions (i.e. a log-normal distribution). For each of these genus ranges, we generated higher taxonomic ranks applying the same simulation approach as for genera and subsequently merged these two data sets. Again, we imitated the number of higher taxonomical ranks from observed data. Each observation in the generated dataset was then binned to one of 80 geological stages. We subsequently applied the same data processing to the simulated ranges as for the observed data including the calculation of short-term and long-term temperature trends for each genus based on our climate proxy data.

Our simulations thus had random (i.e. independent from temperature) LADs and FADs but non-random numbers of higher taxonomic ranks and durations and were linked to our climate proxy data. Finally, we extracted 900 data sets with number of observations ranging from 1000 to 30000 by sampling from the simulated data set with 3000 observations (with replacement). We then applied the GLMMs to these data sets and stored the results. For each data set, we calculated 10 models. Subsequently, we quantified the change in intrinsic extinction risk of each model based on the simulated extinction signal and observed climate proxy data. We repeated this step 100 times for each data set. The simulations hence captured intrinsic biases of both climate proxy data and our applied model structure. We then used the range of variation for the simulated models for significance testing (Fig. 3).

\section{Robustness testing}

Our simulations have shown that a minimum number of 1000 observations are needed to produce statistically robust results (Extended Data 4). We therefore did not apply any 
subsampling method or sampling standardization to the observed data, as reducing the number of observations used in our analysis could increase type II errors. Further, GLMMs do not depend on perfect time continuous data record ${ }^{19}$ and are thus suitable for the imperfect nature of deep-time biotic data used in our analysis. Subsampling all our data would hence only increase statistical uncertainty without improving our analysis. Nevertheless, we tested for a systematic bias in our results by applying two subsampling methods to two fossil clades which had a sufficient number of genera (bivalves, reptiles). Subsampling did not alter results for these two clades, as model comparisons for subsampled data still indicated an improved model performance when a long-term temperature perspective is included (Extended Data 6). As expected, subsampling increased statistical uncertainty resulting in lower differences between AIC values.

We further tested if autocorrelation between the extinction signal and climate proxy data could bias our results (Extended Data 7). For this, we generated autocorrelated random time series for both extinction and climate proxy data and grouped them in a similar structure as our empirical data. We then processed these autocorrelated data sets using the same cleaning protocol as for our empirical data and applied our GLMM model structure to extract the simulated change in extinction risk for autocorrelated data. We did this for 900 data sets with directly correlated extinction/ climate time series (red noise) and for 900 data sets with inversely correlated time series (blue noise). The intensity of autocorrelation for both red and blue noise differed for each simulated data set. Results are within the same range as our null model with a mean change in extinction risk of 0 for red noise and 0.001 for blue noise (mean change of null model $=-0.001$ ). This shows that our model framework accounts for autocorrelation of the underlying data, and that our null model simulation is appropriate to evaluate the probability of obtaining values as extreme as our empirical models. Additional to this, we tested for serial autocorrelation within each final empirical model. We simulated 
residuals for each model and calculated the Durbin-Watson statistic for temporal autocorrelation $^{55}$. No model showed values below 1 or above 3 for the Durbin-Watson statistic, indicating low serial autocorrelation throughout our analysis (Extended Data 7).

Further robustness testing included assessing the impact of mass extinctions on the observed effect. We fitted GLMMs on all fossil groups and all stages and compared these with models excluding stages where the big five mass extinctions occurred (End-Ordovician, Late Devonian, End-Permian, End-Triassic, and End-Cretaceous). Model comparison was based on the conditional coefficient for determination (pseudo- ${ }^{2}$ ) for $G L M M s^{56}$. Models without mass extinctions showed a slightly increased pseudo- $\mathrm{R}^{2}$, indicating that the effect of palaeoclimate interactions on extinction risk is more severe during background extinction events (Extended Data 8).

Data and code availability: All scripts and data used to conduct analyses are available at https://github.com/Ischi94/pal-int-extinction.

\section{Figure legends:}

Fig. 1: Schematic of analytical protocol. (a) For each geological stage, we calculated the magnitude in temperature change compared to the previous stage $\left(\Delta \mathrm{T}_{\text {change }}\right)$ and long-term temperature trends with varying duration $\left(\Delta \mathrm{T}_{\text {Trend }}\right)$. The extinction/survival signal from fossil range data (b), where 1 depicts the extinction of a genus, was combined with the palaeotemperature information using generalised linear mixed effect models (c). The figure illustrates this for one time slice illustrated by the dotted line. The implemented mixed effect models however integrate all time slices in one joint model. (d) In a last step, we quantified the effect of palaeoclimate interaction on intrinsic extinction risk for each palaeoclimate interaction using the results of our models. We subsequently applied the same model structure to simulated fossil data and compared them to our results for statistical inference.

Fig. 2: Model comparison. Model performance of traditional models (change only) was compared to model performance taking palaeoclimate interaction into account (change \& trend). Model performance was evaluated using Akaike's information criterion (AIC). The figure shows the proportional change in AIC of the traditional model compared to the performance of the model including palaeoclimate interactions, for each individual clade. Values above zero (blue dots) show a model improvement for palaeoclimate interactions, and values below zero (red points) a model deterioration. 
Fig. 3: Change in extinction risk due to palaeoclimate interaction of all studied fossil clades. Red data points show change in extinction risk of fossil taxa after warmingwarming palaeoclimate interaction compared to all antagonistic interactions of short-term warming. Blue points show change in extinction risk after cooling-cooling interaction compared to antagonistic interactions of short-term cooling. Points are placed at the median of results and error bars denote $95 \%$ Wald confidence intervals as estimated by Wilcoxon rank sum tests (see Methods). Grey points and confidence intervals demarcate insignificant results based on simulated null models and F statistics, and the grey shaded rectangle shows the range of simulated null models (see Methods).

Fig. 4: Change in extinction risk of fossil clades related to median duration. Red points show change in extinction risk of fossil taxa with warming-warming palaeoclimate interaction. Blue points show change in extinction risk with cooling-cooling interaction. Grey area depicts the $95 \%$ confidence interval of the regression slope. Trend line and R2 value are based on univariate linear regression across all points and significance, indicated by the asterisks $(\mathrm{P}<0.01)$, is based on F statistics.

Fig. 5: Palaeoclimate interactions through the Late Cretaceous and Cenozoic and potential implications for future climate changes. Moderate extinction rates during the Paleocene-Eocene Thermal Maximum (PETM) hyperthermal could be explained by a long-term cooling trend throughout the Late Cretaceous, as cooling-warming interaction potentially mitigates extinction risk. Contrarily, high extinction rates throughout the Late Eocene-Oligocene Cooling (LEOC) could be explained by a previous long-term cooling trend, reinforcing extinction risk. Current palaeontologically informed models did not consider long-term climate trends, which potentially overestimates extinction risk of modern taxa due to current climate change (cooling-warming interaction). Future temperature predictions are taken from the IPCC for surface air temperature ${ }^{57}$ and illustrated by the dotted line. Colours show the direction of temperature trends for both long-term trajectories and short-term change, where red illustrates warming and blue cooling.

\section{References}

1. Molinos, J. G. et al. Climate velocity and the future global redistribution of marine biodiversity. Nature Climate Change 6, 83 (2016).

2. Pecl, G. T. et al. Biodiversity redistribution under climate change: Impacts on ecosystems and human well-being. Science (New York, N.Y.) 355, eaai9214 (2017).

3. Brook, B. W. \& Alroy, J. Pattern, process, inference and prediction in extinction biology (The Royal Society, 2017).

4. Barnosky, A. D. et al. Has the Earth’s sixth mass extinction already arrived? Nature 471, 51 (2011).

5. Thomas, C. D. et al. Extinction risk from climate change. Nature 427, 145 (2004).

6. Urban, M. C. Accelerating extinction risk from climate change. Science (New York, N.Y.) 348, 571-573 (2015).

7. Collins, K. S., Edie, S. M., Hunt, G., Roy, K. \& Jablonski, D. Extinction risk in extant marine species integrating palaeontological and biodistributional data. Proceedings. Biological sciences 285 (2018). 
8. Finnegan, S. et al. Paleontological baselines for evaluating extinction risk in the modern oceans. Science (New York, N.Y.) 348, 567-570 (2015).

9. van Woesik, R. et al. Hosts of the Plio-Pleistocene past reflect modern-day coral vulnerability. Proceedings of the Royal Society B: Biological Sciences 279, 2448-2456 (2012).

10. Harnik, P. G. et al. Extinctions in ancient and modern seas. Trends in Ecology \& Evolution 27, 608-617 (2012).

11. Kiessling, W. \& Kocsis, Á. T. Adding fossil occupancy trajectories to the assessment of modern extinction risk. Biology letters 12, 20150813 (2016).

12. Calosi, P., Putnam, H. M., Twitchett, R. J. \& Vermandele, F. Marine metazoan modern mass extinction: improving predictions by integrating fossil, modern, and physiological data. Annual review of marine science 11, 369-390 (2019).

13. Wiens, J. J. \& Graham, C. H. Niche conservatism: integrating evolution, ecology, and conservation biology. Annu. Rev. Ecol. Evol. Syst. 36, 519-539 (2005).

14. Beaugrand, G. Theoretical basis for predicting climate-induced abrupt shifts in the oceans. Philosophical Transactions of the Royal Society B: Biological Sciences 370, 20130264 (2015).

15. Lord, J. P., Barry, J. P. \& Graves, D. Impact of climate change on direct and indirect species interactions. Marine Ecology Progress Series 571, 1-11 (2017).

16. Bond, D. P. G. \& Grasby, S. E. On the causes of mass extinctions. Palaeogeography, Palaeoclimatology, Palaeoecology 478, 3-29 (2017).

17. Penn, J. L., Deutsch, C., Payne, J. L. \& Sperling, E. A. Temperature-dependent hypoxia explains biogeography and severity of end-Permian marine mass extinction. Science (New York, N.Y.) 362 (2018).

18. Reddin, C. J., Nätscher, P. S., Kocsis, Á. T., Pörtner, H.-O. \& Kiessling, W. Marine clade sensitivities to climate change conform across timescales. Nature Climate Change 10, 249-253 (2020).

19. Bolker, B. M. et al. Generalized linear mixed models: a practical guide for ecology and evolution. Trends in Ecology \& Evolution 24, 127-135 (2009).

20. Burnham, K. P. \& Anderson, D. R. Model selection and multimodel inference. A practical information-theoretic approach. 2nd ed. (Springer, New York, NY, 2010).

21. McKinney, M. L. Extinction vulnerability and selectivity: combining ecological and paleontological views. Annual Review of Ecology and Systematics 28, 495-516 (1997).

22. Foote, M., Crampton, J. S., Beu, A. G. \& Cooper, R. A. On the bidirectional relationship between geographic range and taxonomic duration. Paleobiology 34, 421-433 (2008).

23. Payne, J. L., Truebe, S., Nützel, A. \& Chang, E. T. Local and global abundance associated with extinction risk in late Paleozoic and early Mesozoic gastropods. Paleobiology 37, 616-632 (2011).

24. Harnik, P. G. Direct and indirect effects of biological factors on extinction risk in fossil bivalves. Proceedings of the National Academy of Sciences 108, 13594-13599 (2011). 
25. Harnik, P. G., Simpson, C. \& Payne, J. L. Long-term differences in extinction risk among the seven forms of rarity. Proceedings of the Royal Society B: Biological Sciences 279, 4969-4976 (2012).

26. Hopkins, M. J., Simpson, C. \& Kiessling, W. Differential niche dynamics among major marine invertebrate clades. Ecology Letters 17, 314-323 (2014).

27. Svenning, J.-C. \& Skov, F. Limited filling of the potential range in European tree species. Ecology Letters 7, 565-573 (2004).

28. Normand, S. et al. Postglacial migration supplements climate in determining plant species ranges in Europe. Proceedings of the Royal Society B: Biological Sciences 278, 3644 3653 (2011).

29. Stigall, A. L. When and how do species achieve niche stability over long time scales? Ecography 37, 1123-1132 (2014).

30. Steinbauer, M. J. et al. Biogeographic ranges do not support niche theory in radiating Canary Island plant clades. Global Ecol Biogeogr 25, 792-804 (2016).

31. Foster, G. L., Hull, P., Lunt, D. J. \& Zachos, J. C. Placing our current 'hyperthermal'in the context of rapid climate change in our geological past (The Royal Society Publishing, 2018).

32. Leckie, R. M., Bralower, T. J. \& Cashman, R. Oceanic anoxic events and plankton evolution: Biotic response to tectonic forcing during the mid-Cretaceous.

Paleoceanography 17, 13-1-13-29 (2002).

33. Coxall, H. K. \& Pearson, P. N. The Eocene-Oligocene transition. Deep Time Perspectives on Climate Change: Marrying the Signal From Computer Models and Biological Proxies, 351-387 (2007).

34. Ritterbush, K. A. \& Foote, M. Association between geographic range and initial survival of Mesozoic marine animal genera: circumventing the confounding effects of temporal and taxonomic heterogeneity. Paleobiology 43, 209-223 (2017).

35. Stigall, A. L. Analysing links between biogeography, niche stability and speciation: the impact of complex feedbacks on macroevolutionary patterns. Palaeontology 56, 12251238 (2013).

36. BouDagher-Fadel, M. K. Evolution and geological significance of larger benthic foraminifera (UCL Press, London, 2018).

37. Reddin, C. J., Kocsis, Á. T. \& Kiessling, W. Marine invertebrate migrations trace climate change over 450 million years. Global Ecol Biogeogr 27, 704-713 (2018).

38. Valentine, J. W. Temporal bias in extinctions among taxonomic categories. Journal of Paleontology, 549-552 (1974).

39. Kocsis, Á. T., Reddin, C. J., Alroy, J. \& Kiessling, W. The r package divDyn for quantifying diversity dynamics using fossil sampling data. Methods Ecol Evol 10, 735743; 10.1111/2041-210X.13161 (2019).

40. Gradstein, F. M., Ogg, J. G., Schmitz, M. \& Ogg, G. The geologic time scale 2012 (elsevier, 2012). 
41. Foote, M. Origination and extinction components of taxonomic diversity: general problems. Paleobiology 26, 74-102 (2000).

42. Veizer, J. \& Prokoph, A. Temperatures and oxygen isotopic composition of Phanerozoic oceans. Earth-Science Reviews 146, 92-104 (2015).

43. Song, H., Wignall, P. B., Song, H., Dai, X. \& Chu, D. Seawater temperature and dissolved oxygen over the past 500 million years. Journal of Earth Science 30, 236-243 (2019).

44. Grossman, E. L. Applying oxygen isotope paleothermometry in deep time. The Paleontological Society Papers 18, 39-68 (2012).

45. Henkes, G. A. et al. Temperature evolution and the oxygen isotope composition of Phanerozoic oceans from carbonate clumped isotope thermometry. Earth and Planetary Science Letters 490, 40-50 (2018).

46. Ryb, U. \& Eiler, J. M. Oxygen isotope composition of the Phanerozoic ocean and a possible solution to the dolomite problem. Proceedings of the National Academy of Sciences 115, 6602-6607 (2018).

47. R Core Team. R: A Language and Environment for Statistical Computing (R Foundation for Statistical Computing, Vienna, Austria, 2019).

48. Bates, D., Mächler, M., Bolker, B. \& Walker, S. Fitting Linear Mixed-Effects Models Using lme4. J. Stat. Soft. 67; 10.18637/jss.v067.i01 (2015).

49. Wickham, H. ggplot2: elegant graphics for data analysis (Springer, 2016).

50. Quené, H. \& van den Bergh, H. Examples of mixed-effects modeling with crossed random effects and with binomial data. Journal of Memory and Language 59, 413-425 (2008).

51. Malik, W. A., Marco-Llorca, C., Berendzen, K. \& Piepho, H.-P. Choice of link and variance function for generalized linear mixed models: a case study with binomial response in proteomics. Communications in Statistics-Theory and Methods, 1-20 (2019).

52. Zuur, A., Ieno, E. N., Walker, N., Saveliev, A. A. \& Smith, G. M. Mixed effects models and extensions in ecology with $R$ (Springer Science \& Business Media, 2009).

53. Fox, G. A., Negrete-Yankelevich, S. \& Sosa, V. J. Ecological statistics: contemporary theory and application (Oxford University Press, USA, 2015).

54. Lieberman, M. D. \& Cunningham, W. A. Type I and Type II error concerns in fMRI research: re-balancing the scale. Social cognitive and affective neuroscience 4, 423-428 (2009).

55. Durbin, J. \& Watson, G. S. Testing for serial correlation in least squares regression. III. Biometrika 58, 1-19 (1971).

56. Nakagawa, S., Johnson, P. C. D. \& Schielzeth, H. The coefficient of determination R 2 and intra-class correlation coefficient from generalized linear mixed-effects models revisited and expanded. Journal of the Royal Society Interface 14, 20170213 (2017).

57. Intergovernmental Panel on Climate Change. Global Warming of $1.5^{\circ} \mathrm{C}$ : An IPCC Special Report on the Impacts of Global Warming of $1.5^{\circ} \mathrm{C}$ Above Pre-industrial Levels and Related Global Greenhouse Gas Emission Pathways, in the Context of Strengthening the Global Response to the Threat of Climate Change, Sustainable Development, and Efforts to Eradicate Poverty (Intergovernmental Panel on Climate Change, 2018). 
Acknowledgments: This work was supported by the Deutsche Forschungsgemeinschaft (KI 806/16-1 and STE 2360/2-1) and is embedded in the Research Unit TERSANE (FOR 2332: Temperature-related stressors as a unifying principle in ancient extinctions). M.J.S. acknowledges support by ERC grant 741413 Humans on Planet Earth (HOPE).

Author contributions: G.H.M.: Formal analysis, Investigation, Writing - Original Draft, Visualization/ J.v.D.: Methodology, Writing - Review \& Editing/ W.K.: Resources, Funding acquisition, Writing - Review \& Editing/ M.J.S.: Conceptualization, Supervision, Funding acquisition, Writing - Review \& Editing.

Reporting Summary: Further information on research design is available in the Nature Research Reporting Summary linked to this article.

Competing interests: Authors declare no competing interests.

Materials \& Correspondence: Correspondence and material requests should be addressed to Gregor H. Mathes.

Supplementary Information is available for this paper. 